\title{
Survey on Search Engine Localization and Optimization
}

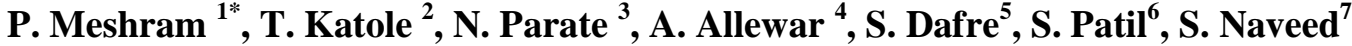 \\ 1* $^{*}$ Dept. of CSE, Priyadarshini J.L. College of Engg.,(Rashtrasant Tukadoji Maharaj Nagpur University), Nagpur, India \\ ${ }^{2}$ Dept. of CSE, Priyadarshini J.L. College of Engg.,(Rashtrasant Tukadoji Maharaj Nagpur University), Nagpur, India \\ ${ }^{3}$ Dept. of CSE, Priyadarshini J.L. College of Engg.,(Rashtrasant Tukadoji Maharaj Nagpur University), Nagpur, India \\ ${ }^{4}$ Dept. of CSE, Priyadarshini J.L. College of Engg.,(Rashtrasant Tukadoji Maharaj Nagpur University), Nagpur, India \\ ${ }^{5}$ Dept. of CSE, Priyadarshini J.L. College of Engg.,(Rashtrasant Tukadoji Maharaj Nagpur University), Nagpur, India \\ ${ }^{6}$ Dept. of CSE, Priyadarshini J.L. College of Engg.,(Rashtrasant Tukadoji Maharaj Nagpur University), Nagpur, India \\ ${ }^{7}$ Dept. of CSE, Priyadarshini J.L. College of Engg.,(Rashtrasant Tukadoji Maharaj Nagpur University), Nagpur, India
}

*Corresponding Author: meshram.pranay@gmail.com,Tel: +91-7020959472

Available online at: www.isroset.org

Received 26/Dec/2017, Revised 10/Jan/2018, Accepted 25/Jan/2018, Online 28/Feb/2018

\begin{abstract}
User get bulk of data whenever he/she search for a particular topic on internet which is not useful for user due to this lots of time is wasted of user. This application which will provide an optimized result for search query according to the user behavior and his previous history. Search engine optimization is a strategical technique to take a web document in top search results of a search engine. Also, localization is a part which will provide notifications of the nearby places, along with the range filter, as per the requirement of the customer. Google is most user friendly Search Engine proved for the Indian users which gives user oriented results. In addition, most of other search engines uses Google search pattern so we have concentrated on it. So, if a page is optimized in Google it is optimized for most of the search engines. This research is based on reviewing various practices that are used to make websites search engine friendly.
\end{abstract}

Keywords - Search Engine Localization; Search engine Optimization; White Hat SEO; Black Hat SEO; Location-based Services.

\section{INTRODUCTION}

Nowadays on internet there is enormous content which made it difficult to find relevant information on a subject. Methods to retrieve such information have become particularly important. So search engine becomes an integral part of everyone's life to search information. We depend on search engines to provide us right information at right time. To satisfy needs of the user search engine must filter and find most allowable information parallel a user query and it should show that information to the user. If search engine decently criticize quality and importance of pages and gives high quality pages to the user then "Search Engine Bias" will not be a significant problem. But unfortunately, page quality is very hard to count in real life. Search engine like Yahoo or Google mainly depend on page rank to measure quality of page. Higher page rank value show website is quite popular in order to score a higher rank in search engine result many website promotion techniques are used by website designers. To promote that website in search engine result, optimizers of that search engine analyses the results and depending on that it show the result and give information about which optimization techniques of search engine are used by website.

Search engine optimization is the method of optimizing any website in such a way that a search engine ranks your website higher than millions of others competing in the same niche. Basically it is about making any website rank higher on popular search engines like Yahoo! and Bing. This process is helpful in driving relevant and organic traffic to your website [1].

Search engine optimizers use optimization techniques which follow search engine guidelines are called white hat SEO techniques. Black hat SEO techniques are used by optimizer of search engine for development of web page which does not follow the rules and policies of search engine.

Search Engine Localization is a location-based service that provides whole information about geographical regions and different places around the world and it is used to get the information about their current location and to process it to get more important information near to that location. It will work on location related and option aware favour this 
system. With the help of A-GPS and GPS system in a phone and through web services using GPRS, HSPA, LTE can implement on android smart phones to provide value added services. Various services provided by Search Engine Localization are as follows:

- Traveling from one destination to another navigation services are used and it offers direction for drivers, walkers, and people of public transportation.

- Individual track portion for a set of GPS coordinates is given by nearest road services which share up to 100 GPS points and gives the nearby road portion for each point. The points which get passed should not need to be branch of a continuous track.

- Search Engine Localization will provide Automatic notification of nearby locations as reported by the geopositions of the user. This app is also helpful for finding out nearby location such as hotel, petrol, pump, etc.

The organization of the paper is as follow, Section I contains the introduction of Search Engine Localization \& Optimization, Section II contain the related work for the Search Engine Localization \& Optimization, Section III contain the proposed system of Search Engine Localization \& Optimization, Section IV concludes research work with future directions.

\section{RELATED WORK}

Joeran Beel [2] in his article he proposed the concept of Academic Search Engine Optimization to optimize literature of scholar for academic websites. The article covers work related that is done mostly in the territory of search engine optimization for Website and defines ASEO and analyze it to search engine optimization for Website. An outline of ranking algorithms for academic search engines is provided in general, just like an outline of Google Scholar's ranking algorithm. It does not give detail how writer or publisher will optimize their Websites and archives for academic search engines.

Vinit Kumar Gunjan [3] in his paper he has discussed that search engine optimization is the idea of boosting the striking of a page by common means i.e., Unrewarded search conclusion. Website go through evolution to make our keywords effectively interact with different search engines. It is done by optimizers it target local search, image and video search. Optimization a page will change contents \& codes of HTML in order to boost its importance to distinct keywords and appropriate indexing in search engines.

J Bao, Y. Zheng [4] in his article he proposed a system for "Location Based And Preference Aware Recommender" which give detail about the nearby places which is within the nearby range with the acknowledge of both user preferences, which are automatically learned from user previous history and current location. Social opinions, which are extracted from the previous location histories of the local experts. As a user can only visit a limited number of locations. The main problem arise when people want to travel new cities and it also become quite challenging. This system only help people to travel not only near their living areas but also to a city that is new to them.

\section{Proposed System}

We are providing an application in which search engine optimization will optimize the user input query and will provide optimized result as per the user behavior. And search engine localization will help the user to find out the nearby location such as hotels, hospitals etc. using scalable technique.

Now the next is Optimization which is the key component of the project. Optimization is as per the user behavior. As per the user requirement the search engine will rank the website or the search result on the top. Optimization will be done according to the number of hits i.e. frequency count on a particular website. More the frequency count more above the website will go on the top of the page. Also the frequency count of the keywords are being maintained so as to recommend or suggest a particular website to a user.

For optimization we are using White Hat SEO. White Hat SEO includes using of keywords and the frequency of occurrence of a particular keyword in a search query. Also it does keyword analysis and link building to improve the link popularity for a link to be on the top of a page. White Hat is also called an Ethical SEO.

Thus the project is the combination of Optimization and Localization and overcomes the drawbacks of the earlier SEOs in the following ways:

- This project depend on the number count of an appropriate keyword which increases the ranking of a particular website briskly. Earlier it was based on the cumulative work adding to the reputation of any website hence the impact building was slower.

- This project also offers Localization as a part along with the optimization technique. This will help in building relevant traffic to a particular website as well as supporting and generating the lead in sales 


\section{CONCLUSION}

The research paper provides the brief idea about Search Engine Optimization and Localization and their working. It also illustrates the search engine optimization techniques like Black Hat SEO and White Hat SEO. We have studied all the existing system found that they are using $A^{*}$ Algorithm the main disadvantage of $\mathrm{A}^{*}$ Algorithm is that if any obstacle encountered then it return back to the start point so to overcome that we are using Dijkstra's Algorithm it always give the quickest and the fastest track for Localization. Also we are using White hat optimization technique for query optimization so that the query will always be according to user behavior. As a future dimension to this research, I intend to develop an effective and accurate system for search engine optimization for obtaining a higher rank for the websites in the search results.

\section{REFERENCES}

[1] Neha Sahu, Richa Chhabra," Review on Search Engine Optimization", Volume 6, Issue 6, June (2016).

[2] Joeran Beel, Bela Gipp, and Erik Wilde,"Academic Search Engine Optimization (ASEO): Optimizing Scholarly Literature for Google Scholar" 41 (2): 176-190, January 2010.

[3] Vinit Kumar Gunjan1, Pooja2, Monika Kumari3 ,Dr Amit Kumar4 ,Dr (col.) Allam appa rao5," Search engine optimization with Google " IJCSI International Journal of Computer Science Issues, Vol. 9, Issue 1, No 3, January 2012.

[4] J. Bao, Y. Zheng and M. F. Mokbel, "LocationBased and Preference-Aware Recommendation" Proceedings of the 20th Inter- national Conference on Advances in Geographic Information Systems SIGSPATIAL, Redondo Beach, 6-9 November 2012, pp. 199-208.

\section{Authors Profile}

Mr. Pranay Meshram pursed

Bachelor of Engineering from

Sant Gadge Baba Amravati

University,Amravati in 2006

and Master of Technology from

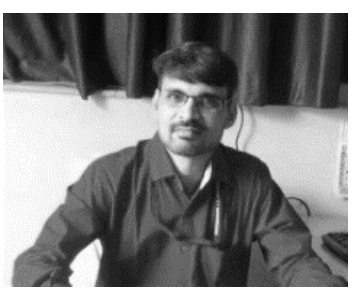

Rashtrasant Tukadoji Maharaj Nagpur University in year 2012. He is currently pursuing Ph.D. and currently working as Assistant Professor in Department of Computer Science \& Engineering,PJLCOE,Nagpur. He is a member of IEEE, a life time member of AMIE since 2008, a life member of the
ISTE since 2014, a life member of IAENG since 2014, a life member of SDIWC since 2014, a life time member of ISOC,a life time member of IACSIT,a member of UACEE. He has published more than 10 research papers in reputed international journals and conferences. His main research work focuses on Selective encryption Algorithms, Cryptography Algorithms, Network Security. He has 12 years of teaching experience.

Tejashree Katole is pursuing her bachelor of engineering in Computer Science \& Engineering from Priyadarshini J.L of Engineering, Rashtrasant Tukadoji

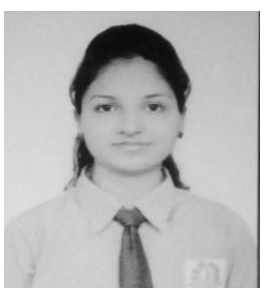

Maharaj Nagpur University, Nagpur-440009, India

Nandini Parate is pursuing her bachelor of engineering in Computer Science \& Engineering from Priyadarshini J.L of Engineering, Rashtrasant Tukadoji Maharaj Nagpur University, Nagpur-440009, India

Ashwini Allewar is pursuing her bachelor of engineering in Computer Science \& Engineering from Priyadarshini J.L of Engineering, Rashtrasant Tukadoji Maharaj Nagpur University, Nagpur-440009, India

Shubham Dafre is pursuing his bachelor of engineering in Computer Science \& Engineering from Priyadarshini J.L of Engineering, Rashtrasant Tukadoji

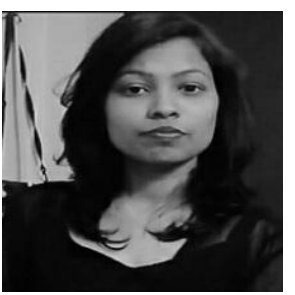

Maharaj Nagpur University, Nagpur-440009, India 


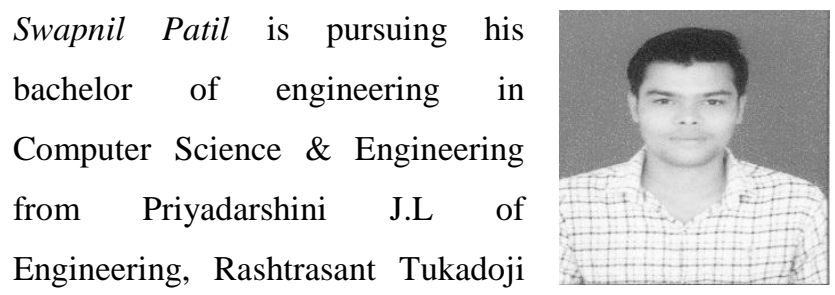

Maharaj Nagpur University, Nagpur-440009, India

Sheikh Naveed is pursuing his bachelor of engineering in Computer Science \& Engineering from Priyadarshini J.L of Engineering, Rashtrasant Tukadoji

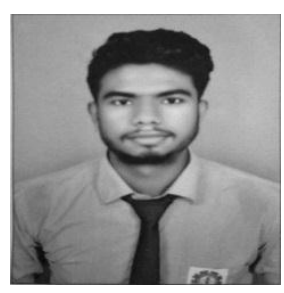

Maharaj Nagpur University, Nagpur-440009, India 